\title{
The effects of metformin on gut microbiota and the immune system as research frontiers
}

\author{
Michael Pollak ${ }^{1}$
}

Received: 18 May 2017 / Accepted: 15 June 2017 /Published online: 2 August 2017

(C) Springer-Verlag GmbH Germany 2017

\begin{abstract}
Recent studies have revealed that metformin influences gut microbiota and the immune system although neither is a classic target of the drug. This research has revealed complexity not previously appreciated, and opened new research directions. The extent to which immunomodulatory effects and actions on the microbiota are related to each other and account for effects on host energy metabolism remains to be determined. These sites of action may be relevant not only to the efficacy of metformin for its established use in type 2 diabetes, but also to proposed novel indications in oncology and other diseases.
\end{abstract}

Keywords Diabetes $\cdot$ Metformin $\cdot$ Microbiome $\cdot$ Microbiota immunity cancer
Abbreviations
AMPK AMP-activated protein kinase
GLP-1 Glucagon-like peptide 1
PD-1 Programmed cell death protein 1

Electronic supplementary material The online version of this article (doi:10.1007/s00125-017-4352-x) contains a slide of the figure for download, which is available to authorised users.

Michael Pollak

michael.pollak@mcgill.ca

1 Division of Cancer Prevention, Department of Oncology, McGill University, Montreal, QC H3T 1E2, Canada

\section{Background}

The history of the development of metformin leading to its current status as the most widely used compound for the treatment of type 2 diabetes mellitus was not one of rational drug design. Rather, serendipity and clues arising from remedies used by mediaeval herbalists played important roles. Elucidation of the rather unexpected molecular mechanisms of action followed much later, and remains incomplete. As detailed elsewhere [1], mediaeval treatments for polyuria included extracts of the plant Galega officinalis. These extracts were presumably beneficial for a subset of patients complaining of excess urination, specifically those suffering from the disease we now know as type 2 diabetes, which when poorly controlled is a cause of polyuria.

When treatments for diabetes were being sought in the mid 20th century, examination of Galega officinalis extracts suggested that guanidine and galegine were likely to be the active ingredients. It was demonstrated experimentally that these could reduce the hyperglycaemia, glycosuria and polyuria found in of type 2 diabetes, but clinical administration of galegine was limited by toxicity. This led to the screening of biguanides (guanidine derivatives) for ability to lower blood glucose levels. Of the numerous biguanides examined, metformin (dimethylbiguanide) appeared to have the best therapeutic index. It was initially introduced into clinical practice in Europe in the 1950s, but only became widely used in North America in the 1990s. Despite the subsequent development of several different classes of drug for type 2 diabetes, metformin remains the mainstay of treatment because of its safety and efficacy, and is recommended by most authorities as the first line treatment when drug therapy is indicated. It is available from many generic manufacturers, and is among the least expensive drugs for diabetes. At a cost of less than a dollar a day, it is often the only affordable glucose-lowering drug for 
patients in the developing world, where the epidemic of type 2 diabetes is severe. However, its increasing use is not simply a function of its availability and price: efficacy and safety are key factors.

\section{Molecular targets}

The mechanisms of action of metformin have been reviewed extensively [2-5]. At the molecular level, many targets have been studied (for examples, [6, 7]) but most studies have focused on respiratory complex I of the electron transport chain $[8,9]$. The precise interaction between the tiny biguanide molecule and the huge multi-protein complex has not been established, although ongoing research is addressing that question. Many earlier laboratory studies (for example [10]) of the mechanism of action of metformin were somewhat imprecise as the major read-out was simply the decline in oxygen consumption by whole cells. However, recent work has shown activity of biguanides, including metformin, on isolated mitochondria [11] and on isolated complex I [9], providing more specificity. While oxidative phosphorylation is clearly inhibited by many biguanides, there are interesting complexities regarding determinants of intracellular and mitochondrial concentration and accumulation at the site of action, all of which interact to determine activity, and also lead to differences between specific biguanide molecules; while metformin is used for diabetes, proguanil is used in treatment of malaria, and others are antiseptics [9].

The older literature (reviewed in $[2,4,5]$ attributed the cellular effects of complex I inhibition mainly to ATP deficiency arising from reduced oxidative phosphorylation: this leads to direct consequences for ATP-requiring reactions, but also to indirect consequences arising from the activation of AMP-activated protein kinase (AMPK), which reprograms cellular energy metabolism such that energy consumption is diminished and ATP production is maximised. There are many downstream consequences of AMPK activation, with key examples being inhibition of fatty acid synthesis and the mechanistic target of rapamycin (mTOR) signalling network [12], both of which lead to reduced energy consumption. More recently, it has been recognised that complex I inhibition has additional consequences related to accumulation of NADH relative to $\mathrm{NAD}^{+}$, which of course would also be expected to influence cellular biochemistry. One example of this is the inhibitory effect of biguanides on aspartate synthesis [13].

At the whole organism level, the actions of metformin depend on the extent to which the drug accumulates in different organs. Pharmacokinetic factors, as well as differences between cell lineages in the expression of the cell surface transporters that import the drug, imply that the drug accumulates in a heterogeneous manner between organs following oral administration [14]. The liver is a classic site of action of metformin and it is probably exposed to a higher level of the drug than other organs, following oral administration, because the portal circulation would be expected to have a higher drug concentration than the systemic circulation. As a consequence of energetic stress secondary to inhibition of oxidative phosphorylation following metformin exposure, the drug inhibits hepatocyte gluconeogenesis, resulting in reduced hepatic glucose output. This ameliorates the hyperglycaemia found in type 2 diabetes and secondarily reduces hyperinsulinaemia. There is also evidence that metformin reduces oxidative phosphorylation in muscle [15], which probably leads to compensatory increases in glucose uptake and glycolysis, further contributing to correction of hyperglycaemia.

Recently, there has been increased interest in actions of metformin on the gut microbiota and the immune system, topics that had not previously been investigated in detail despite the widespread use of the drug. As there are relationships among the immune system, the microbiota and energy metabolism at the whole organism level [16-18], effects of metformin on any one of these may influence the others, and contribute to therapeutic effects. In this brief review, recent progress and gaps in knowledge in these areas will be summarised.

\section{Metformin and gut microbiota}

There is convincing evidence $[19,20]$ that metformin exposure induces significant changes in human intestinal microbiota (for example, decreases in abundance of Intestinibacter), and in the gut metabolome (for example, increased butyrate production). These observations stemmed from efforts to confirm and extend prior research (for examples, [21-23]) concerning relationships between microbiota composition, obesity and diabetes. They revealed that certain prior studies (for example, [24]) may have been confounded by the effects of metformin on the gut microbiota. The mechanism by which metformin influences the gut microbiota remains undefined. We have shown that biguanides inhibit bacterial complex I in a manner that is not fundamentally different from effects on mammalian complex I [9]. However, this does not represent conclusive evidence that effects of metformin on the gut microbiota are attributable to inhibition of bacterial complex I leading to inhibition of the electron transport chain. In Caenorhabditis elegans, metformin has been observed to increase lifespan in a manner that is attributable to effects of the drug on folate metabolism within bacteria in the gut, although a specific molecular target for metformin that leads to these alterations remains to be defined [25]. Biguanides have long been known to have antimicrobial activity [26], and some are used as antimalarials and others as antiseptics. The antimalarial mechanisms have been elucidated [9], but the critical targets for antibacterial actions of metformin represent an 
important gap in our knowledge. However, it is likely that the effects of metformin in the complex ecosystem of the gut lumen are heterogeneous among bacterial species, leading to the observed metformin-induced changes in microbiota composition.

There is additional complexity in relation to factors that influence the gut metformin concentration following oral ingestion [27]. It is noteworthy that metformin concentration in the gut lumen can be over 100-fold that present in serum, implying that mechanisms of action requiring high millimolar concentrations that are not achieved systemically [28] may be relevant to effects on the microbiota. Rates of systemic absorption and intestinal transit time affect the level of metformin the microbiota are exposed to: oral bioavailability is around $30-60 \%$ and approximately $50 \%$ of an ingested dose is found in the faeces, although both these factors exhibit considerable person-toperson variability. Hence the degree to which the intestinal flora are altered by the drug is affected by host factors.

In view of evidence (reviewed in [18]) that person-toperson variability in gut microbiota composition can be related to risk for obesity and diabetes, it is of interest to determine the extent to which intestinal actions of the drug, which include but are not confined to alterations of gut microbiota, account for therapeutic benefits. Evidence (reviewed in [16, 29]) that blood glucose-lowering effects of a poorly absorbed metformin formulation can be demonstrated suggests that at least a portion of the therapeutic benefit of metformin is attributable to effects within the gastrointestinal system. How can a drug acting only within the gut affect systemic carbohydrate metabolism? Specific microbiome profiles are associated with increased glucose intolerance [30]. Alterations in gut microbiota can influence the gut metabolome in a manner that affects the intestinal output of butyrate [24] and acetate [31]. These are important examples of gut-derived metabolites that can influence host insulin resistance, and thus glycaemic control. Gut microbiota can also influence glucagon-like peptide I (GLP-1) physiology: for example, a reduction of Lactobacilli is associated with GLP-1 resistance related to alterations in enteric neuron nitric oxide production [32].

It is important to recognise, however, that metformin may also influence systemic carbohydrate metabolism by gastroenteric mechanisms that do not directly involve gut microbiota. These may include alterations in bile acid physiology, enteric hormones (reviewed in [27]), and effects on duodenal AMPK signalling that contribute to suppression of hepatic gluconeogenesis [33]. While recent work has provided clear evidence that metformin alters gut microbiota, the classic mechanisms of action such as inhibition of gluconeogenesis also remain relevant to its efficacy in treatment of type 2 diabetes, and further research is needed to estimate the proportion of the benefit of metformin attributable to changes in gut microbiota. It would not be surprising if this varies considerably between metformin-treated individuals.

\section{Metformin and the immune system}

Clinical experience has not provided evidence for major effects of metformin on immune function, as neither immunodeficiency nor increased autoimmunity have been reported as common adverse effects of administration of the drug. Although no detailed clinical studies examining influences of metformin on more subtle aspects of immune function have been carried out, laboratory studies [34-45] have provided considerable evidence for a variety of immunomodulatory effects.

Work in the field of immunometabolism has shown that AMPK influences T cell effector responses [34]. Therefore, the prior body of evidence that metformin inhibits oxidative phosphorylation, leading to AMPK activation, provides a plausible basis for effects of the drug on immune function. Indeed, metformin has been used as a tool to upregulate AMPK to demonstrate the relevance of this energy sensor to $\mathrm{T}$ cell memory [35]. While the notion that metformin may influence immune function as a consequence of altering energy metabolism in the many cell lineages that comprise the immune system is straightforward, it is unclear what subpopulations of immune cells are most affected by the drug, and therefore what the net effect on immune function would be in different clinical contexts. Recent results in tumour immunology provide examples of enhanced immune function as a consequence of metformin exposure [36-38], while studies in other contexts, including tuberculosis and multiple sclerosis, suggest anti-inflammatory actions [40-45].

A conceptually direct experimental approach compared the efficacy of metformin as an anti-neoplastic drug in intact vs immunodeficient mice [36], and showed greatly increased anti-tumour activity of the drug in mice with a functional immune system, where metformin increased the number of CD8-positive tumour-infiltrating lymphocytes in an AMPKdependent fashion. A separate laboratory study also provides evidence for enhancement of anti-tumour immunity by the biguanide phenformin [37]. However, in this model, the biguanide increases immune function by inhibiting granulocytic myeloid-derived suppressor cells, rather than by enhancing immune effector cells. The proposed mechanism involves induction of reactive oxygen species by the drug, with secondarily reduced suppressor function. Of potential clinical relevance is the finding in this study of phenformin enhancement of the tumour-inhibitory effect of anti-programmed cell death protein 1 (PD-1) antibody therapy on growth of the Braf(V600E)/Pten null melanoma mouse model, such that the combination of phenformin and the antibody cooperatively induces $\mathrm{CD}^{+} \mathrm{T}$ cell infiltration. Another study [38] also documented enhancement of PD-1 blockade as a cancer immunotherapy, but attributed this to a mechanism involving reduction of tumour hypoxia by the biguanide, which in turn was attributed to reduced oxygen consumption secondary to 
inhibition of oxidative phosphorylation. Effects of metformin on immunologic memory have been associated with enhanced efficacy of an anti-tumour vaccine in a laboratory model, with a significant effect on host survival [35]. Both the clinical relevance and mechanistic details of these studies remain to be determined. Among other areas of investigation, it will be important to clarify dose-response aspects of putative actions of metformin as an enhancer of anti-tumour immunity, and to study the expression on immune effector and suppressor cells of cell surface transporters (such as organic cation transporter 1 [OCT-1]) required for metformin import.

Although the studies cited above raise the possibility that immunomodulatory actions of biguanides may contribute to the antineoplastic activity seen in certain experimental models, there are important caveats. First, both older (reviewed in [4]) and more recent [46] models demonstrate tumour growth inhibition by biguanides in immune-deficient mice, suggesting that mechanisms other than immune modulation operate. Second, the relevance of all the preclinical models to patient care remains to be established, despite a plausible theoretical rationale and encouraging preclinical data [47] as the first randomised, placebo-controlled trial of metformin in cancer treatment failed to demonstrate a benefit of the drug in treatment of pancreatic cancer [48].

Separate research indicates that there are contexts in which metformin acts as an anti-inflammatory or immunosuppressive agent. We have observed strong experimental evidence that metformin can act to suppress inflammation associated with senescence [40], and there is also convincing evidence from in vivo and in vitro models that the drug suppresses cancer-related inflammation [41]. In both cases, NF- KB activation is reduced, with concomitant reductions in secretion of inflammatory cytokines. However, the precise molecular target of metformin that leads to inhibition of NF- $\mathrm{KB}$ remains to be defined. Inhibition of the innate immune response to fungal infection by metformin in an AMPK-dependent manner has been observed in model systems [42], but the clinical relevance of these observations also remains to be determined.

In multiple sclerosis, an early clinical study [43] concluding that metformin lessens central nervous system inflammation and improves disease outcomes deserves confirmation. Experimental evidence [44] suggests that metformin can influence CD4 T cell metabolism and reduce autoimmunity in a manner that improves experimental systemic lupus. An additional study [45] suggests that the mechanism involved in reduction of autoimmunity requires AMPK activation, which is achieved as a consequence of inhibition of oxidative phosphorylation in B cells. Metformin also reduces inflammation associated with pulmonary tuberculosis [49], but further work is needed to clarify the relative importance of effects on the host vs the Mycobacterium itself.

How can one reconcile the impressive and diverse effects of metformin on the immune system found in experimental models, on the one hand, with clinical experience on the other, where immunomodulatory effects of the drug are not obvious? Most clinical studies of immune function among metformin users (for example, [50]) have used relatively simple endpoints, such as C-
Fig. 1 Overview of systemic and gastrointestinal actions of metformin, and how they may translate into therapeutic benefits for type 2 diabetes and other conditions, as well as have implications for the use of other biguanides

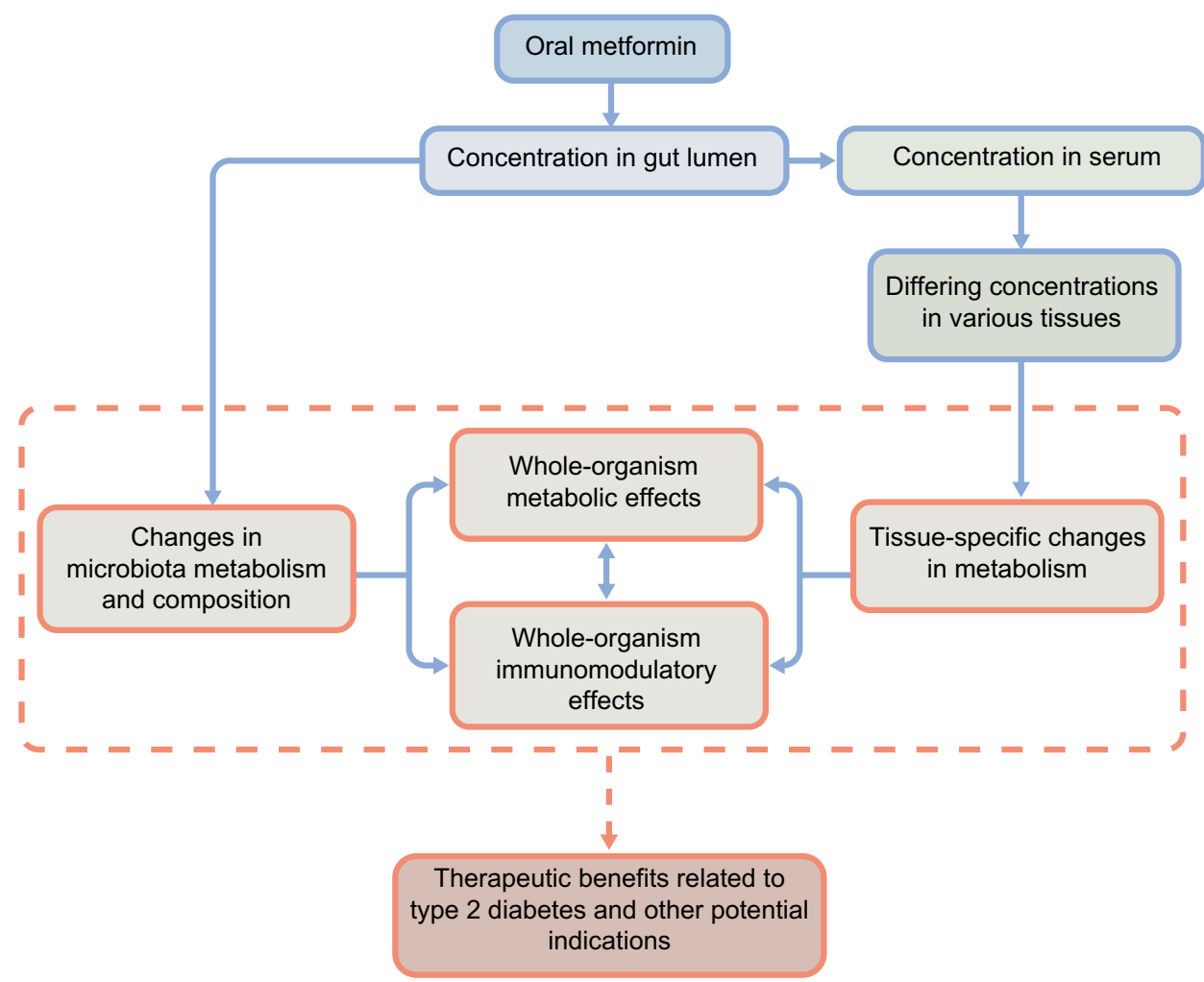


reactive protein. While decreases in this non-specific inflammatory marker have been observed, the relevance of this finding in relation to clinical benefits of the drug is uncertain. While some may conclude that the consequences of metformin administration in the model systems reviewed are clinically irrelevant, it is possible that the effects of metformin on immune function vary in complex ways depending on the baseline status of the immune system. For example, if certain autoimmune diseases are associated with upregulation of cell surface metformin transport molecules on immune effector cells, the consequences of metformin exposure may be different in these disease states from those in typical individuals with type 2 diabetes treated with metformin. Thus, further studies are needed to determine whether or not the early experimental evidence represents a valuable clue to clinically relevant properties of metformin.

\section{Conclusion}

Metformin not only has a colourful history, but more importantly provides broad clinical benefits to tens of millions of people for its established use in the treatment of type 2 diabetes. Ongoing laboratory studies, initiated decades after its approval for diabetes treatment, continue to refine our understanding of its mechanisms of action. As shown in Fig. 1, the drug has ample opportunity to exert effects on the gastrointestinal tract following oral administration (including effects on gut microbiota) as gut lumen concentrations are considerably higher than circulating concentrations. These gastrointestinal effects can have both local and systemic consequences, but certainly do not exclude any effects of absorbed metformin on classic target organs such as the liver, or on more recently proposed target cell lineages such as those of the immune system or neoplastic tissue. The effects of metformin on the microbiome and immune system may be relevant not only to diabetes treatment, but also to proposed novel indications, for example in oncology and ageing [51-53]. In future work, careful attention to whole organism and cellular pharmacokinetics [14] will be crucial to establishing the clinical relevance of laboratory studies.

Acknowledgements R. Lim (Pollak Research Lab, Lady Davis Research Institute/McGill University) contributed to the design of figures and to manuscript editing.

Funding No external funding was used for this work.

Duality of interest The author declares that there is no duality of interest associated with this manuscript.

Contribution statement The author was the sole contributor to this paper.

\section{References}

1. Bailey CJ (2017) Metformin: historical overview. Diabetologia. doi:10.1007/s00125-017-4318-Z

2. Foretz M, Guigas B, Bertrand L, Pollak M, Viollet B (2014) Metformin: from mechanisms of action to therapies. Cell Metab 20:953-966

3. An H, He L (2016) Current understanding of metformin effect on the control of hyperglycemia in diabetes. J Endocrinol 228:R97106

4. Pollak MN (2012) Investigating metformin for cancer prevention and treatment: the end of the beginning. Cancer Discov 2:778-790

5. Pryor R, Cabreiro F (2015) Repurposing metformin: an old drug with new tricks in its binding pockets. Biochem J 471:307-322

6. Kalender A, Selvaraj A, Kim SY et al (2010) Metformin, independent of AMPK, inhibits mTORC1 in a rag GTPase-dependent manner. Cell Metab 11:390-401

7. Madiraju AK, Erion DM, Rahimi Y et al (2014) Metformin suppresses gluconeogenesis by inhibiting mitochondrial glycerophosphate dehydrogenase. Nature 510:542-546

8. Bridges HR, Sirvio VA, Agip AN, Hirst J (2016) Molecular features of biguanides required for targeting of mitochondrial respiratory complex I and activation of AMP-kinase. BMC Biol 14:65

9. Bridges HR, Jones AJ, Pollak MN, Hirst J (2014) Effects of metformin and other biguanides on oxidative phosphorylation in mitochondria. Biochem J 462:475-487

10. Jalling O, Olsen C (1984) The effects of metformin compared to the effects of phenformin on the lactate production and the metabolism of isolated parenchymal rat liver cell. Acta Pharmacol Toxicol 54: 327-332

11. Andrzejewski S, Gravel SP, Pollak M, St-Pierre J (2014) Metformin directly acts on mitochondria to alter cellular bioenergetics. Can Metab 2:12

12. Howell JJ, Hellberg K, Turner M et al (2017) Metformin inhibits hepatic mTORC1 signaling via dose-dependent mechanisms involving AMPK and the TSC complex. Cell Metab 25:463-471

13. Gui DY, Sullivan LB, Luengo A et al (2016) Environment dictates dependence on mitochondrial complex I for $\mathrm{NAD}^{+}$and aspartate production and determines cancer cell sensitivity to metformin. Cell Metab 24:716-727

14. Liang X, Giacomini KM (2017) Transporters involved in metformin pharmacokinetics and treatment response. J Pharm Sci. doi:10. 1016/j.xphs.2017.04.078

15. Wang PY, Li J, Walcott FL et al (2017) Inhibiting mitochondrial respiration prevents cancer in a mouse model of Li-Fraumeni syndrome. J Clin Invest 127:132-136

16. Brunkwall L, Orho-Melander M (2017) The gut microbiome as a target for prevention and treatment of hyperglycaemia in type 2 diabetes: from current human evidence to future possibilities. Diabetologia 60:943-951

17. Zmora N, Bashiardes S, Levy M, Elinav E (2017) The role of the immune system in metabolic health and disease. Cell Metab 25: 506-521

18. Komaroff AL (2017) The microbiome and risk for obesity and diabetes. JAMA 317:355-356

19. Forslund K, Hildebrand F, Nielsen Tet al (2015) Disentangling type 2 diabetes and metformin treatment signatures in the human gut microbiota. Nature 528:262-266

20. Wu H, Esteve E, Tremaroli V et al (2017) Metformin alters the gut microbiome of individuals with treatment-naive type 2 diabetes, contributing to the therapeutic effects of the drug. Nat Med. doi: $10.1038 / \mathrm{nm} .4345$

21. Turnbaugh PJ, Ley RE, Mahowald MA, Magrini V, Mardis ER, Gordon JI (2006) An obesity-associated gut microbiome with increased capacity for energy harvest. Nature 444:1027-1031 
22. David LA, Maurice CF, Carmody RN et al (2014) Diet rapidly and reproducibly alters the human gut microbiome. Nature 505:559563

23. Karlsson FH, Tremaroli V, Nookaew I et al (2013) Gut metagenome in European women with normal, impaired and diabetic glucose control. Nature 498:99-103

24. Qin J, Li Y, Cai Z et al (2012) A metagenome-wide association study of gut microbiota in type 2 diabetes. Nature 490:55-60

25. Cabreiro F, Au C, Leung KY et al (2013) Metformin retards aging in $C$. elegans by altering microbial folate and methionine metabolism. Cell 153:228-239

26. Weinberg ED (1968) Antimicrobial activities of biguanides. Ann N Y Acad Sci 148:587-600

27. McCreight LJ, Bailey CJ, Pearson ER (2016) Metformin and the gastrointestinal tract. Diabetologia 59:426-435

28. Chandel NS, Avizonis D, Reczek CR et al (2016) Are metformin doses used in murine cancer models clinically relevant? Cell Metab 23:569-570

29. Buse JB, DeFronzo RA, Rosenstock J et al (2016) The primary glucose-lowering effect of metformin resides in the gut, not the circulation: results from short-term pharmacokinetic and 12-week dose-ranging studies. Diabetes Care 39:198-205

30. Pedersen HK, Gudmundsdottir V, Nielsen HB et al (2016) Human gut microbes impact host serum metabolome and insulin sensitivity. Nature 535:376-381

31. Perry RJ, Peng L, Barry NA et al (2016) Acetate mediates a microbiome-brain-beta-cell axis to promote metabolic syndrome. Nature 534:213-217

32. Grasset E, Puel A, Charpentier J et al (2017) A specific gut microbiota dysbiosis of type 2 diabetic mice induces GLP-1 resistance through an enteric NO-dependent and gut-brain axis mechanism. Cell Metab 25:1075-1090.e1075

33. Duca FA, Cote CD, Rasmussen BA et al (2015) Metformin activates a duodenal AMPK-dependent pathway to lower hepatic glucose production in rats. Nat Med 21:506-511

34. Blagih J, Coulombe F, Vincent EE et al (2015) The energy sensor AMPK regulates $T$ cell metabolic adaptation and effector responses in vivo. Immunity 42:41-54

35. Pearce EL, Walsh MC, Cejas PJ et al (2009) Enhancing CD8 T-cell memory by modulating fatty acid metabolism. Nature 460:103-107

36. Eikawa S, Nishida M, Mizukami S, Yamazaki C, Nakayama E, Udono H (2015) Immune-mediated antitumor effect by type 2 diabetes drug, metformin. Proc Natl Acad Sci U S A 112:1809-1814

37. Kim SH,LiM, Trousil Setal(2017) Phenformin inhibits myeloid-derived suppressor cells and enhances the anti-tumor activity of PD-1 blockade in melanoma. J Invest Dermatol. doi:10.1016/j.jid.2017.03.033

38. Scharping NE, Menk AV, Whetstone RD, Zeng X, Delgoffe GM (2017) Efficacy of PD-1 blockade is potentiated by metformininduced reduction of tumor hypoxia. Cancer Immunol Res 5:9-16
39. Cameron AR, Morrison VL, Levin D et al (2016) Antiinflammatory effects of metformin irrespective of diabetes status. Circ Res 119:652-665

40. Moiseeva O, Deschenes-Simard X, St-Germain E et al (2013) Metformin inhibits the senescence-associated secretory phenotype by interfering with IKK/NF- $\mathrm{KB}$ activation. Aging Cell 12:489-498

41. Hirsch HA, Iliopoulos D, Struhl K (2013) Metformin inhibits the inflammatory response associated with cellular transformation and cancer stem cell growth. Proc Natl Acad Sci U S A 110:972-977

42. Cheng SC, Quintin J, Cramer RA et al (2014) mTOR- and HIF-1 $\alpha$ mediated aerobic glycolysis as metabolic basis for trained immunity. Science 345:1250684

43. Negrotto L, Farez MF, Correale J (2016) Immunologic effects of metformin and pioglitazone treatment on metabolic syndrome and multiple sclerosis. JAMA Neurol 73:520-528

44. Yin $\mathrm{Y}$, Choi SC, $\mathrm{Xu} \mathrm{Z}$ et al (2015) Normalization of $\mathrm{CD}^{+} \mathrm{T}$ cell metabolism reverses lupus. Sci Transl Med 7:274ra218

45. Lee SY, Moon SJ, Kim EK et al (2017) Metformin suppresses systemic autoimmunity in Roquin ${ }^{\mathrm{san} / \mathrm{san}}$ mice through inhibiting B cell differentiation into plasma cells via regulation of AMPK/mTOR/STAT3. J Immunol 198:2661-2670

46. Rajeshkumar NV, Yabuuchi S, Pai S et al (2017) Treatment of pancreatic cancer patient-derived xenograft panel with metabolic inhibitors reveals efficacy of phenformin. Clin Cancer Res doi: 10.1158/1078-0432.CCR-17-1115/

47. Pollak M (2012) Metformin and pancreatic cancer: a clue requiring investigation. Clin Cancer Res 18:2723-2725

48. Kordes S, Pollak MN, Zwinderman AH et al (2015) Metformin in patients with advanced pancreatic cancer: a double-blind, randomised, placebo-controlled phase 2 trial. Lancet Oncol 16: 839-847

49. Singhal A, Jie L, Kumar P et al (2014) Metformin as adjunct antituberculosis therapy. Sci Transl Med 6:263ra159

50. Goldberg RB, Temprosa MG, Mather KJ, Orchard TJ, Kitabchi AE, Watson KE (2014) Lifestyle and metformin interventions have a durable effect to lower CRP and tPA levels in the diabetes prevention program except in those who develop diabetes. Diabetes Care 37:2253-2260

51. Barzilai N, Crandall JP, Kritchevsky SB, Espeland MA (2016) Metformin as a tool to target aging. Cell Metab 23:1060-1065

52. Martin-Montalvo A, Mercken EM, Mitchell SJ et al (2013) Metformin improves healthspan and lifespan in mice. Nat Commun 4:2192

53. Klil-Drori AJ, Azoulay L, Pollak MN (2017) Cancer, obesity, diabetes, and antidiabetic drugs: is the fog clearing? Nat Rev Clin Oncol 14:85-99 